\title{
Using a 3D metamaterial to enhance surface wave propagation in HF band
}

\author{
Quentin Herbette* $^{* \dagger}$, Nicolas Bourey ${ }^{\dagger}$, Michel Menelle*, Muriel Darces ${ }^{\dagger}$, Stéphane Saillant*, Marc Hélier ${ }^{\dagger}$ \\ *DEMR, ONERA, Université Paris Saclay F-91123 Palaiseau, France, quentin.herbette@ onera.fr* †Sorbonne \\ Université, CNRS, Laboratoire de Génie Electrique et Electronique de Paris, 75252, Paris, France \\ Université Paris-Saclay, CentraleSupélec, CNRS, Laboratoire de Génie Electrique et Electronique de Paris, \\ 91192, Gif-sur-Yvette, France.
}

\begin{abstract}
This paper deals with the characterization of a 3D metamaterial structure to enhance the surface wave radiation of transmission antennas used with $\mathrm{HF}$ surface wave radars. The innovation is to use a corrugated surface with a negative effective permittivity to launch surface waves. In order to verify the predicted behaviour of this metamaterial antenna, near-field measurements were carried out at scale 1:100 in UHF band and scale 1:1 in HF band. Measurements at $1 \mathrm{GHz}$ were performed on a small scale mock-up in a semi-anechoic chamber. The HF characterization was carried out outdoors thanks to an on-board measuring system on a Unmanned Aerial Vehicle (UAV) specially developed for this testing. The results are presented and allow to confirm the expected radiation of the system.
\end{abstract}

Index Terms-HF Antennas, Near-Field Measurement, Metamaterial.

\section{INTRODUCTION}

ONERA has been deploying surface wave radars for more than a decade. These radars, operating in the HF band (between 3 and $30 \mathrm{MHz}$ ) and using surface waves, are interesting low-cost solutions for the detection of targets beyond the radio electric horizon. These radars use antennas similar to those of sky wave radars, which are not optimized for surface wave radiation [1]. As a result, a project aimed at increasing the surface wave radiation of these antennas has been initiated. The study began by researching the conditions for launching a powerful surface wave. It was shown that a metamaterial of negative permittivity $\left(\varepsilon_{r r}=-10\right.$ typically) would fulfill the requirement [2]. Simulations and experiments carried out at $1 \mathrm{GHz}$ (scale 1:100) have shown that a corrugated surface of negative permittivity allows the excitation of a larger surface wave. A full scale HF metamaterial was then developed. This structure has the following dimensions $\left(21 \times 5 \times 2.5 \mathrm{~m}^{3}\right)$ and is shown in figure 1. It has been built in stainless steel. The structure is installed on a ground plane and connected to it.

In this figure, we also see the measuring system embedded aboard the UAV which is used to characterize the system outdoors. This original system allows to measure the near electric field in amplitude and phase [3].

In section II, the design of the metamaterial is introduced. In section III, the UHF measuring system is described and the results obtained at $1 \mathrm{GHz}$ are presented. In section IV, the experimental results on the full scale HF metamaterial are shown.

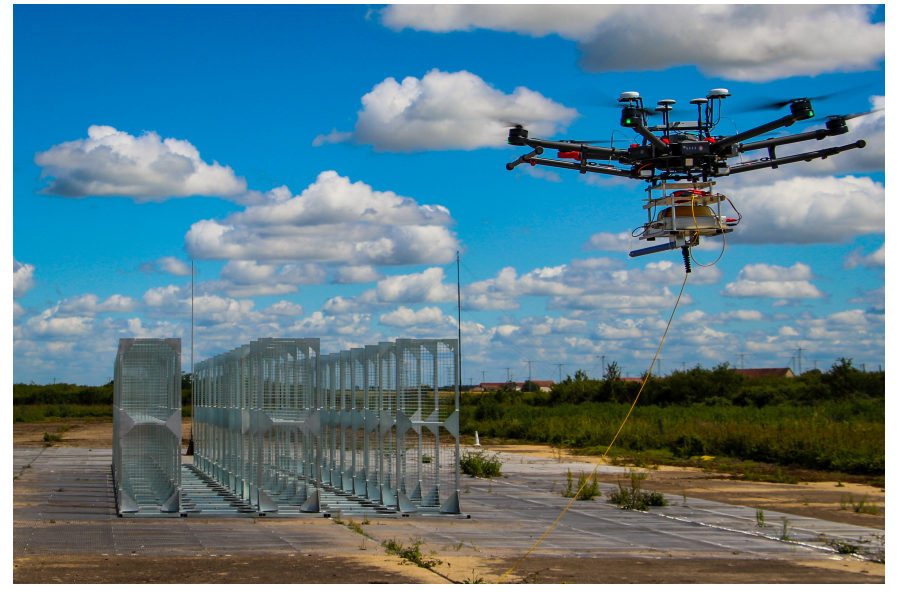

Fig. 1: 3D metamaterial used in HF band and the near field measurement system embedded aboard a UAV

\section{DEFINITION OF THE METAMATERIAL}

In 2011, it was shown, using a Kistovich decomposition of the field [4] that only interface with negative permittivity can propagate a sole surface wave. To fulfil the propagation constraints, it is necessary to have a relative permittivity lower than $\varepsilon_{r r}=-10$ and a conductivity as low as possible [2]. In order to materialize a metamaterial with such properties, a periodic structure is used. That kind of periodic structures has been widely studied and can be found in various domains of application. The most usual structures are the corrugated surfaces, mushroom surfaces and uni-planar surfaces.

Those structures have a frequency band gap in which the surface wave cannot propagate. In the passband, the surface wave can be more or less strong and confined, depending on the properties of the periodic structure.

The chosen periodic structure is a corrugated surface which is able to propagate a "Spoof Surface Plasmon Polaritons", i.e. a TM surface wave concentrated at the interface. The corrugated surface is a grounded dielectric slab with included vertical parallel metallic walls. Considering infinite length along axes $x$ and $y$ the obtained periodical structure shows a motif which parameters are : $d$ the length, $a$ the width of each groove, $h$ the depth of each groove. Such a structure can be observed in figure 2 for which the excitation source is provided by a monopole antenna (not represented here). 


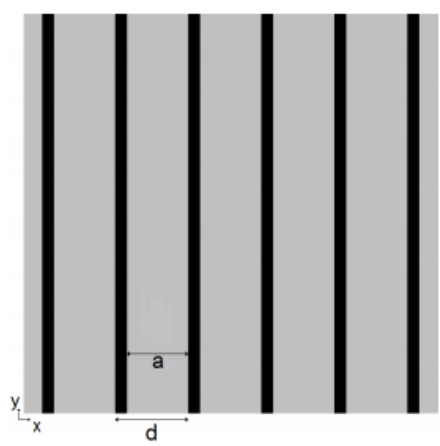

(a)

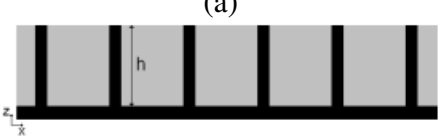

(b)

Fig. 2: Geometry of a corrugated surface (a) Top view (b) Cross view

\section{SMALL-SCALE MEASUREMENT}

In order to verify the expected radiation of the structure and the presence of a wave confined to the interface, a reduced scale structure (1:100) was built. This structure working at $1 \mathrm{GHz}$ is shown in figure 3 and exhibits a difference with the geometry illustrated in figure 2 . The metal walls are replaced by panels, which reduces the volume of material needed to build the structure, and the weight of it. This new structure has been designed thanks to a geometric optimization of the panels constituting the metamaterial structure (the optimization procedure is not the subject of this communication).

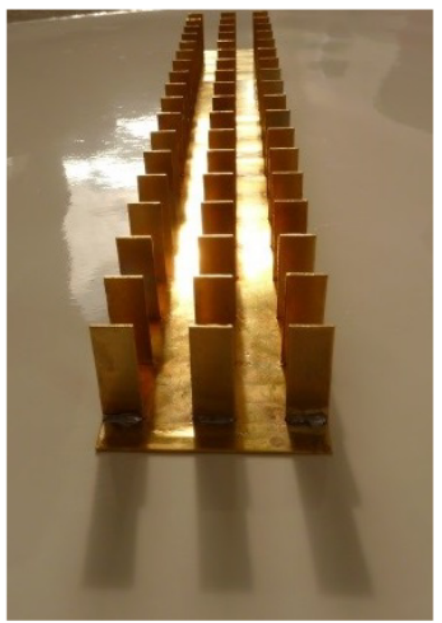

Fig. 3: Metamaterial structure designed at $1 \mathrm{GHz}$

An experimental bench for near-field characterization of UHF antennas has been developed [5]. This bench relies on a near-field probe mounted on motorized rails which allow the movement of the probe along 3 axes. In order to characterize the radiation of the monopole exciting the metamaterial surface, the field is measured on a closed cylindrical virtual surface encompassing the whole structure. A near field to far field transformation is then applied to calculate the field at long distance [6]. A similar process is performed for a monopole without the metamaterial structure. A relative gain (in $\mathrm{dB}$ ) is finally calculated as:

$$
\text { Gain }=20 \log \left(\frac{\mid \text { Emeta }_{Z} \mid}{\left|E_{Z}\right|}\right)
$$

Where Emet $_{Z}$ is the electric field radiated by a monopole with the structure and $E_{Z}$ is the electric field radiated by the monopole only. As it can be seen in figure 4 , a final gain of 10 $\mathrm{dB}$ in the direction of interest ( $x$ axis), at ground level and at a distance $750 \mathrm{~mm}(2.5 \lambda)$ from the source can be expected.

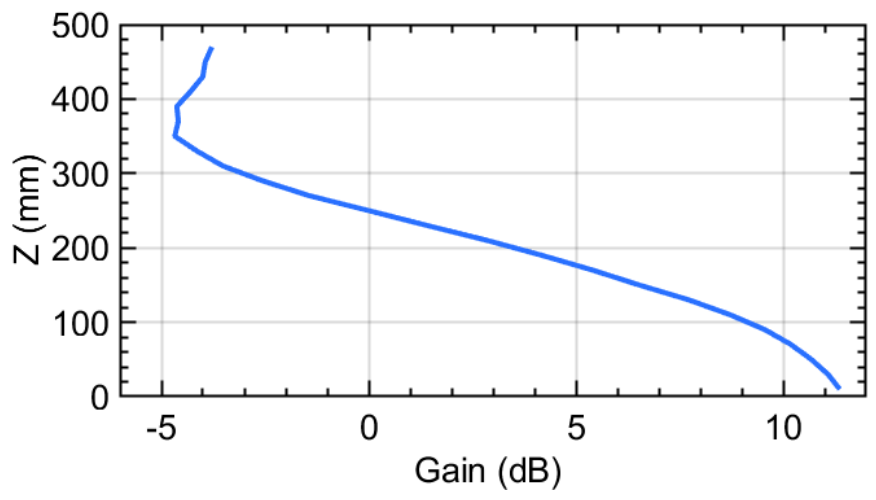

Fig. 4: Results obtained at $1 \mathrm{GHz}$ at ground level and at a distance of $750 \mathrm{~mm}(2.5 \lambda)$ from the source

\section{HF BAND METAMATERIAL MEASUREMENT}

A dedicated measurement device has been developed and assembled to check the expected radiation of the metamaterial at scale $1(15 \mathrm{MHz})$. This system is based on a near electric field probe loaded onto a UAV. The embedded measuring system is a EFS-105 near electric field probe designed by Enprobe (sensitivity $<30 \mu \mathrm{V} /(\mathrm{m} \sqrt{\mathrm{Hz}})$ ).

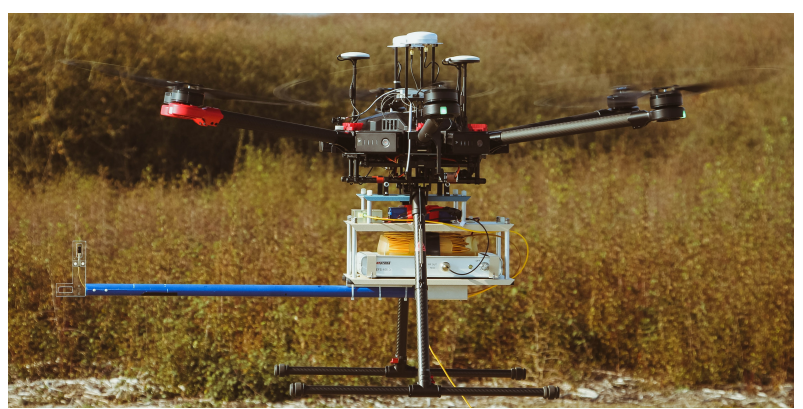

Fig. 5: Measurement system aboard the UAV

The final purpose is to determine the radiation pattern of the transmission system. Taking into account the dimensions of the structure, it should have been impossible to perform the characterization in an anechoic chamber. Moreover, in the HF band, the environment has a strong influence on the radiation of the antennas. So in order to characterize the transmission system, and considering the large far field distance, it is necessary to perform an outdoor near field characterization 
to determine the far field via a suited transformation [6]. For that purpose, it is required to know the electric field in amplitude and phase. The phase is difficult to obtain because a synchronization between the transmission and the reception signals is required. At $15 \mathrm{MHz}$, a precision on the phase shift of $10^{\circ}$ corresponds to a precision of $2 \mathrm{~ns}$ on the time shift. This difficulty is the major constraint in the development of the measurement system.

In order to solve the synchronization issue, the measurement system has been equipped with an optical fiber. It is used to bring back, at ground level, the information acquired by the near electric field probe. This allows to use the same clock for the digitization of the transmitted and received signals. This solution is concurrently deployed by other researchers [7] willing to use a UAV to determinate antennas radiation patterns. Optical fiber is lightweight and consequently has a low impact on the weight of the payload. The optical fiber is insensitive to radio frequency which makes it resilient to any disturbances on the electric field information. A GNSS RTK positioning system was integrated aboard the UAV. It provides an accuracy of less than $10 \mathrm{~cm}$ on the drone positioning. Last, to reduce coupling effects between the measured antennas and the UAV, it is mandatory to move the probe aside from the drone. Thanks to electromagnetic simulation the needed distance between the sensor and the drone could be determined. Since the drone manufacturer has not communicated on the properties of the UAV materials, the simulation has been performed assuming the worst case scenario, a perfect electrical conductor (PEC) structure. In order to have a negligible error, it is required to locate the sensor at least at $70 \mathrm{~cm}$ from the UAV. The required distance is obtained by means of a fiberglass tube. The developed measurement system mounted on the UAV can be seen in figure 5 .

A block diagram illustrating the measurement can be seen in figure 6. The signal from the local oscillator propagates to a coupler and a part of it excites the antenna under test. In our case the excitation source was a monopole antenna. Thanks to the onboard electro-optical converter, the information on the near-electric field is converted into a light signal. With the use of a long optical fiber and an optoelectronic converter, it is converted, at ground level, back to an analogical signal. This signal and the emitted one are digitized, using the same clock, by a National Instruments digitizing system. The latter is used as a reference for the phase.

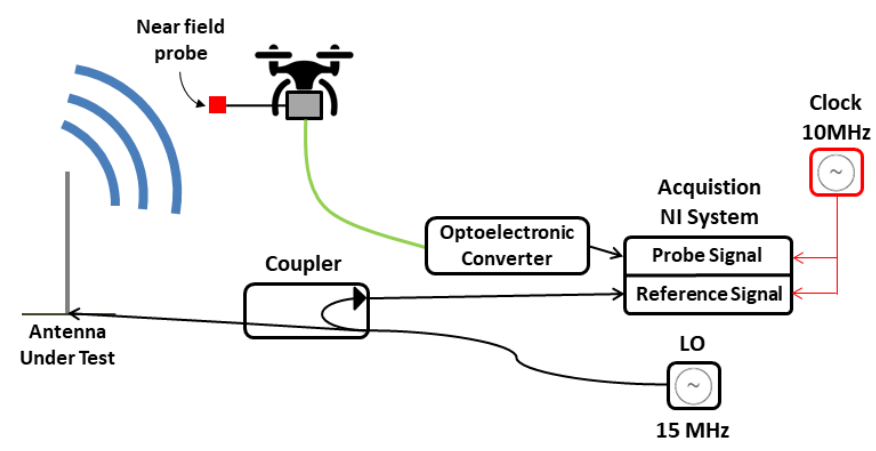

Fig. 6: Block diagram of the antenna measurement
Three types of measurement paths have been carried out. The first one is a test measurement (Fig. 7a) which is a set of horizontal flights above the structure used to check the reproductibility of the results and are considered as reference measurements. The second one is composed of horizontal measurements carried out to map the field above the metamaterial which enable to visualize the surface plasmon (Fig. 7b). Finally, in order to perform the near to far field transformation, it is needed to know the field on a closed virtual surface centered on the transmission system. Figure 7c depicts the vertical and radial lines followed by the UAV to describe a corresponding closed cylinder.

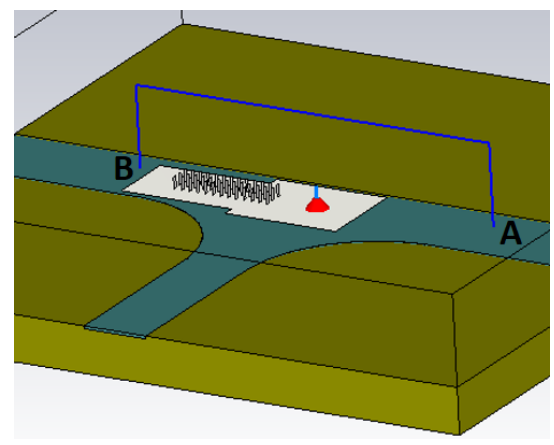

(a)

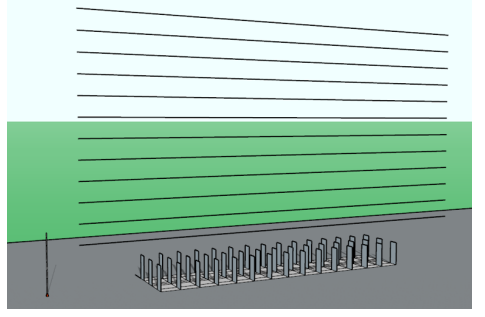

(b)

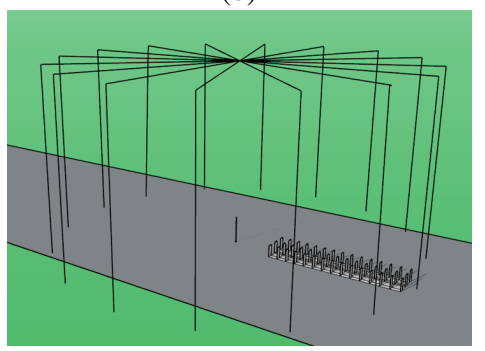

(c)

Fig. 7: The three types of measurement paths

In order to validate the experimental data, the measurement environment has been reproduced in simulation under CST MWS. Also, since the ground on which the transmission structure is located is heterogeneous, it has been modeled using the dielectric characteristics of concrete and soil. These simulations and test measurements made it possible to quickly verify that the transmission system behaves as expected. The blue path $(\mathrm{AB})$ in figure $7 \mathrm{a}$ is the UAV trajectory. These measurements are compared with simulations for which the UAV's flight path was faithfully reproduced. As can be seen in figure 8, there is a good agreement between the two types of results, both in amplitude and phase (point A corresponds to 


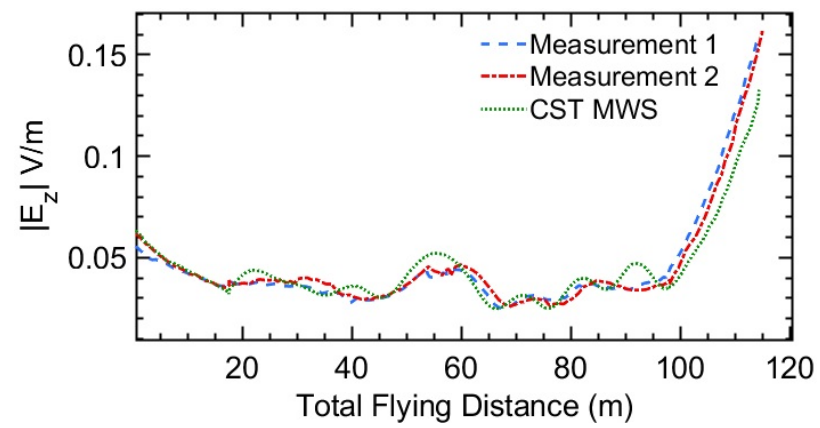

(a)

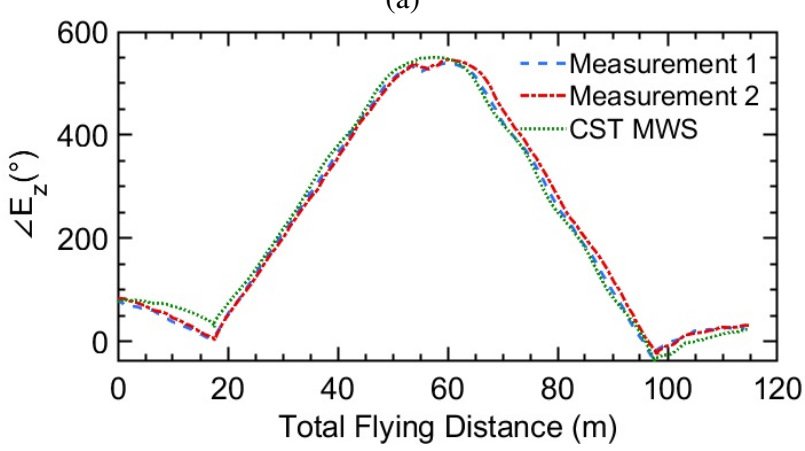

(b)

Fig. 8: Test measurements - Amplitude and phase of the vertical electric field : comparison between measurements and simulations

$x=0 \mathrm{~m}$ and point $\mathrm{B}$ to $x=115 \mathrm{~m}$ ). Similar results could be obtained for the radial component of the electric field. Since the azimuthal component of the field is intrinsically null, it has not been measured.

The second results are those obtained with measurements along horizontal lines. These measurements were carried out for 9 frequencies in order to highlight the bandwidth of the metamaterial structure. The measurements performed at 15 and $18.8 \mathrm{MHz}$ are presented in figure 9a. The figure 9b shows the simulation results obtained in the same conditions. We can see that the measured and simulated distributions of the electric field are very close to each other. The surface plasmon can be observed. Some differences can be explained by the interpolation algorithm used to generate field maps from lines. The mean squared errors for both frequency has been evaluated at $\mathrm{RMSE}_{15 \mathrm{MHz}}=-36.7 \mathrm{dBV} / \mathrm{m}$ and $\mathrm{RMSE}_{18.8 \mathrm{MHz}}=-30.6 \mathrm{dBV} / \mathrm{m}$. At the end, the results concerning the cylindrical measurement for the near to far field transformation will be presented during the conference.

\section{CONCLUSION}

In this communication we have presented a metamaterial structure allowing to generate a strong confined wave. This system was first characterized at $1 \mathrm{GHz}$, which made it possible to determine the expected gain of our transmission system, then in HF band. For that purpose, we have also presented an airborne UAV-based system that can measure the near electric field in amplitude and phase. It was used to verify the expected behaviour of the structure in the HF band. This
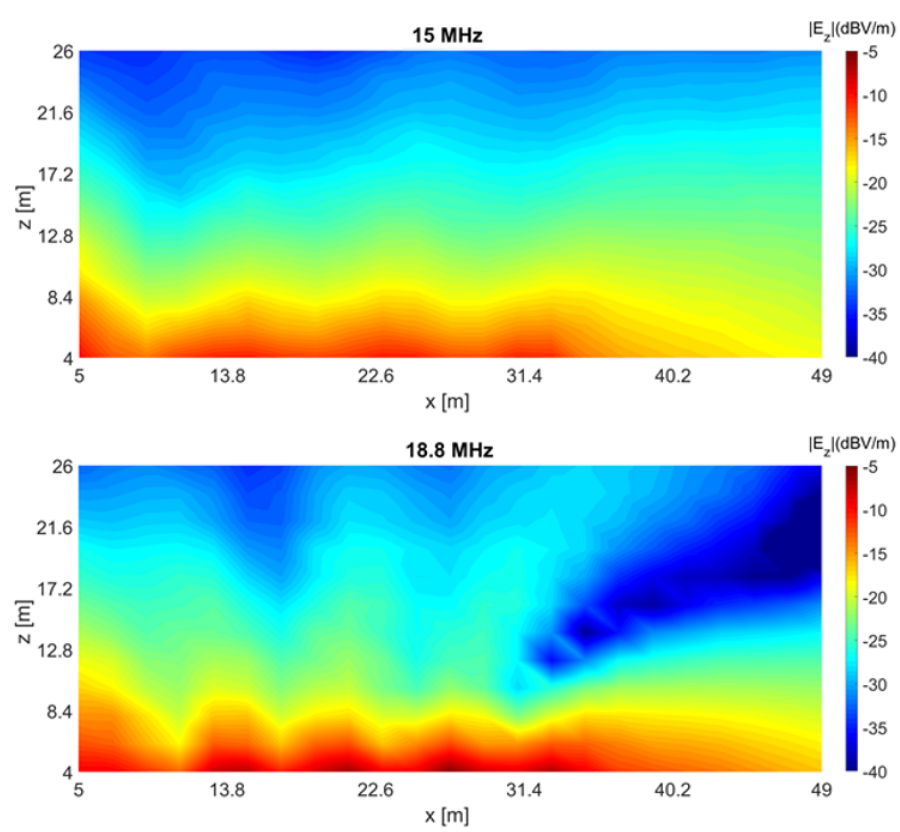

(a)
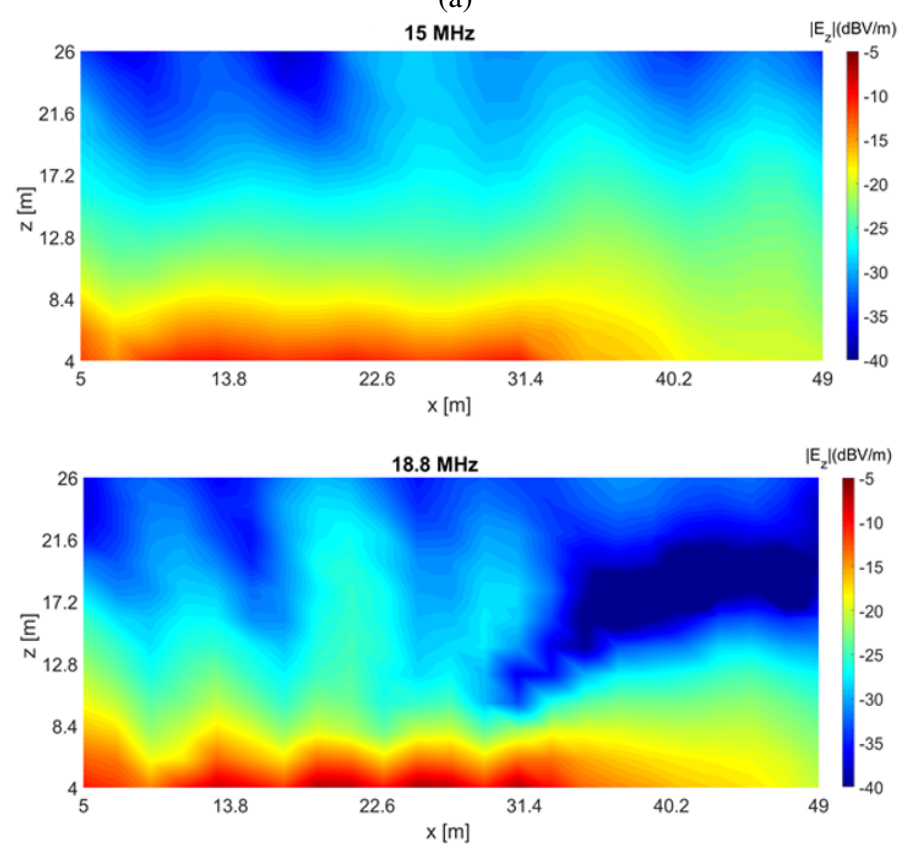

(b)

Fig. 9: Horizontal lines - Amplitude of the vertical electric field : (a) measurements at $15 \mathrm{MHz}$ and $18.8 \mathrm{MHz}$ (b) simulations at $15 \mathrm{MHz}$ and $18.8 \mathrm{MHz}$

system is soon to be deployed in operational conditions, with radar measurements, which will allow an accurate assessment of its effective radiation gain. The results of the measurements and near field to far field transformations will be presented at the conference.

\section{ACKNOWLEDGMENT}

DGA (French Ministry of Defense) is thanked for its contractual support to the RAPID AC3M project for which 
the metamaterial and the airborne system have been designed and developed.

\section{REFERENCES}

[1] F. Jangal and M. Menelle. French HFSWR contribution to the European integrated maritime surveillance system I2C. In IET International Radar Conference 2015, pages 1-5, October 2015.

[2] L. Petrillo, F. Jangal, M. Darces, J.-L. Montmagnon, and M. Hélier. Negative Permittivity Media Able to Propagate a Surface Wave. Progress In Electromagnetics Research, 115:1-10, 2011. Publisher: EMW Publishing.

[3] Q. Herbette, S. Saillant, M. Menelle, B. Urbani, N. Bourey, M. Darces, and M. Hélier. HF Radar antenna near field assessment using a UAV. In 2019 International Radar Conference (RADAR), pages 1-4, September 2019. ISSN: 2640-7736.

[4] Yu V Kistovich. On the possibility of observing surface zenneck waves in the radiation of a source with a small vertical aperture. Journal of Technical Physics, 59(4):16-21, 1989.

[5] N. Bourey, M. Darces, and M. Hélier. In Situ Antenna Far Field Estimation Based on Equivalent Sources. In 2018 USNC-URSI Radio Science Meeting (Joint with AP-S Symposium), pages 105-106, July 2018.

[6] N. Payet, M. Darces, Jean-Louis Montmagnon, Marc Hélier, and Florent Jangal. Near field to far field transformation by using equivalent sources in HF band. In 201215 International Symposium on Antenna Technology and Applied Electromagnetics, pages 1-4, June 2012.

[7] T. Fritzel, R. Strauß, H.-J. Steiner, C. Eisner, and T. Eibert. Introduction into an UAV-based near-field system for in-situ and large-scale antenna measurements (Invited paper). In 2016 IEEE Conference on Antenna Measurements Applications (CAMA), pages 1-3, October 2016. 